\title{
Do carotid MR surface coils affect PET quantification in PET/MR imaging?
}

\author{
Martin J Willemink ${ }^{1 *}$, Mootaz Eldib ${ }^{1}$, Tim Leiner ${ }^{2}$, Zahi A Fayad ${ }^{1}$, Venkatesh Mani ${ }^{1}$ \\ From PSMR 2015: 4th Conference on PET/MR and SPECT/MR \\ La Biodola, Isola d'Elba, Italy. 17-21 May 2015
}

${ }^{1}$ Translational and Molecular Imaging Institute, Icahn School of Medicine at Mount Sinai, New York, NY, USA
To evaluate the effect of surface coils for carotid MR imaging on PET quantification in a clinical simultaneous whole-body PET/MR scanner. A cylindrical phantom was filled with a homogeneous 2L water-FDG mixture at a starting dose of 301.2MBq. Clinical $\mathrm{PET} / \mathrm{MR}$ and PET/CT systems were used to acquire PET-data without a coil (reference standard) and with two carotid MRI coils (Siemens Special Purpose 8-Channel and Machnet 4-Channel Phased Array). PET-signal attenuation was evaluated with Osirix using 51 (PET/MR) and 37 (PET/CT) circular ROIs. Mean and maximum standardized uptake values (SUVs) were quantified for each ROI. Furthermore, SUVs of PET/MR and PET/CT were compared. For validation, a patient was scanned with an injected dose of $407.7 \mathrm{MBq}$ on both a PET/CT and a PET/MR system without a coil and with both coils. PET/MR underestimations were $-2.2 \%$ (Siemens) and $-7.8 \%$ (Machnet) for SUVmean, and $-1.2 \%$ (Siemens) and $-3.3 \%$ (Machnet) for SUVmax, respectively. For $\mathrm{PET} / \mathrm{CT}$, underestimations were $-1.3 \%$ (Siemens) and $-1.4 \%$ (Machnet) for SUVmean and $-0.5 \%$ (both Siemens and Machnet) for SUVmax, respectively using no coil data as reference. Except for PET/CT SUVmax values all differences were significant. SUVs differed significantly between PET/MR and PET/CT with SUVmean values of 0.51-0.55 for PET/MR and 0.68-0.69 for PET/CT, respectively. The patient examination showed that median SUVmean values measured in the carotid arteries decreased from 0.97 without a coil to 0.96 (Siemens) and 0.88 (Machnet). Carotid surface coils do affect attenuation correction in both PET/MR and PET/CT imaging. Furthermore, SUVs differed significantly between PET/MR and PET/CT.

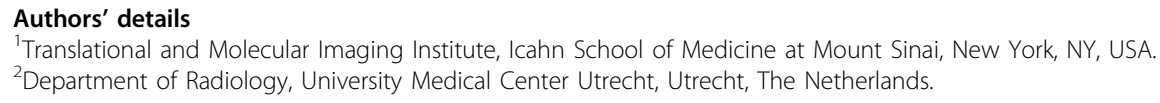

doi:10.1186/2197-7364-2-S1-A34

Cite this article as: Willemink et al: Do carotid MR surface coils affect PET quantification in PET/MR imaging? EJNMMI Physics 2015 2(Suppl 1):A34.

\section{SpringerOpen $^{\odot}$}

(c) 2015 Willemink et al; licensee Springer. This is an Open Access article distributed under the terms of the Creative Commons Attribution License (http://creativecommons.org/licenses/by/4.0), which permits unrestricted use, distribution, and reproduction in any medium, provided the original work is properly cited. 